\title{
Simulation on the Self-Compacting Concrete by an Enhanced Lagrangian Particle Method
}

\author{
Jun Wu, ${ }^{1}$ Xuemei Liu, ${ }^{2}$ Haihua $X u,{ }^{3}$ and Hongjian $\mathrm{Du}^{4}$ \\ ${ }^{1}$ School of Urban Railway Transportation, Shanghai University of Engineering Science, Shanghai 201620, China \\ ${ }^{2}$ School of Civil Engineering and Built Environment, Queensland University of Technology, Brisbane, \\ QLD 4000, Australia \\ ${ }^{3}$ Deepwater Technology, Keppel Offshore \& Marine Technology Centre, Singapore 628130 \\ ${ }^{4}$ Department of Civil and Environmental Engineering, The National University of Singapore, Singapore 117576
}

Correspondence should be addressed to Xuemei Liu; x51.liu@qut.edu.au

Received 10 June 2016; Accepted 14 July 2016

Academic Editor: Robert Cerný

Copyright (C) 2016 Jun Wu et al. This is an open access article distributed under the Creative Commons Attribution License, which permits unrestricted use, distribution, and reproduction in any medium, provided the original work is properly cited.

\begin{abstract}
The industry has embraced self-compacting concrete (SCC) to overcome deficiencies related to consolidation, improve productivity, and enhance safety and quality. Due to the large deformation at the flowing process of SCC, an enhanced Lagrangian particlebased method, Smoothed Particles Hydrodynamics (SPH) method, though first developed to study astrophysics problems, with its exceptional advantages in solving problems involving fragmentation, coalescence, and violent free surface deformation, is developed in this study to simulate the flow of SCC as a non-Newtonian fluid to achieve stable results with satisfactory convergence properties. Navier-Stokes equations and incompressible mass conservation equations are solved as basics. Cross rheological model is used to simulate the shear stress and strain relationship of SCC. Mirror particle method is used for wall boundaries. The improved SPH method is tested by a typical 2D slump flow problem and also applied to L-box test. The capability and results obtained from this method are discussed.
\end{abstract}

\section{Introduction}

Industrialization of construction is the main trend in scientific and technical progress in construction, including the extensive use of prefabricated factory-finished elements and the conversion of production into a mechanized and continuously flowing process of assembly and installation of buildings and structures made of prefabricated assemblies and elements. It will lead to higher-quality materials and more cost-effectiveness and energy efficiency for infrastructure construction. Self-consolidating concrete (SCC) is a thixotropic mixture, created by tailoring different materials and securing excellent deformability and adequate resistance to segregation to insure the exceptional rheology behavior of concrete [1]. The industry has embraced SCC to overcome deficiencies related to consolidation, improve productivity, and enhance safety and quality. To follow up the demanding for the exceptional flowability of SCC to further improve its filling behavior and improve the performance of SCC, it is essential to fully understand its flow behavior and the infilling characteristics. Such understanding could further improve the prediction of the infilling behavior of SCC with the presence of reinforcing steel distributed with high density or formwork in complex and irregular shapes. To achieve such purpose, simulation may serve as an effective tool in the understanding as well as determining the desired rheological performance of SCC.

While simulation is a mature science in homogeneous fluids, simulation of the flow of SCC is difficult because of the need to track the boundaries between the liquid and solid phases. Few previous studies have focused on finding the rheological material model for certain paste or concrete [2-4].

In considering the large deformation nature of SCC flow and great complexity of concrete-infilling behavior, the conventional methods such as discrete element method (DEM) and finite element method (FEM) have two critical drawbacks 
including the limitations in modeling free surfaces and nonguaranteed conservation of mass. The recently developed Smoothed Particles Hydrodynamics (SPH) method offers a glimpse of the future of fresh concrete flow simulation. Although SPH was first developed to study astrophysics problems $[5,6]$, it has exceptional advantages in enabling the simulation of flow and infilling behavior. It has been used to study wave-structure interactions for Newtonian flow [7-9]. It has also been successfully used to simulate non-Newtonian flow such as in metal forming [10], the impacting droplet [11], blood-vessel interaction [12], and Couette flow [13]. In one case, it was employed to simulate the slump of concrete [14]. In comparison with other methods, the SPH method can solve the two issues encountered in DEM and FEM. The SPH method has advantages in solving problems involving fragmentation and coalescence and violent free surface deformation and has the potential to link rheology and the infilling behavior of concrete. Therefore, the particle-based Lagrangian technique-SPH method is a promising method to simulate the flow of SCC as a non-Newtonian.

However, the existing SPH still has limitations when applied to non-Newtonian fluid. Firstly, it cannot measure the velocity divergence and pressure gradient accurately. Secondly, it cannot treat the nonslip boundary condition exactly, which is an issue for SSC. Hence, further enhancement of the existing SPH method will be the focus of this study fluid to achieve accurate and stable results with satisfactory convergence properties. And the improved SPH algorithm is tested by a typical 2D slump flow problem and then applied for an L-box flow problem. The SCC is modeled using Cross rheological model. The capability and results obtained from this method are discussed. The simulation provides reasonable good agreement with available data from the literature. The findings may be of engineering interests in view of the flowability and pressure caused by fresh SCC on structures.

\section{Mathematical Model and SPH Formulation}

2.1. Governing Equation. In the current study, the SCC is assumed as viscous non-Newtonian fluid, which is governed by the Navier-Stokes (NS) equation. The 2D Lagrangian form is $[14,17]$

$$
\begin{aligned}
& \nabla \cdot \overrightarrow{\mathbf{u}}=0, \\
& \frac{D \overrightarrow{\mathbf{u}}}{D t}=-\frac{1}{\rho} \nabla p+\overrightarrow{\mathbf{g}}+\frac{1}{\rho} \nabla \cdot \overrightarrow{\boldsymbol{\tau}},
\end{aligned}
$$

where $\rho$ is fluid density, $t$ is time, $\overrightarrow{\mathbf{u}}=(u, v)$ is velocity vector, $p$ is pressure, $\overrightarrow{\mathbf{g}}=\left(g_{x}, g_{y}\right)$ is gravitational acceleration, $D(\cdot) / D t$ is Lagrangian operator, which is the total time derivative, $\nabla$ is gradient operator, and $\overrightarrow{\boldsymbol{\tau}}$ is shear stress tensor. The shear stress tensor is related to the strain tensor $\vec{\gamma}$ as

$$
\tau_{i j}=\tau_{j i}=\mu_{\mathrm{eff}} \gamma_{i j}
$$

where $\mu_{\text {eff }}$ is effective viscosity, $\tau_{i j}$ and $\gamma_{i j}$ are the components of $\overrightarrow{\boldsymbol{\tau}}$ and $\overrightarrow{\boldsymbol{\gamma}}$, respectively, and $\gamma_{i j}$ is defined as

$$
\gamma_{i j}=\frac{1}{2}\left(\frac{\partial u_{i}}{\partial x_{j}}+\frac{\partial u_{j}}{\partial x_{i}}\right)
$$

For non-Newtonian fluid, the relationship of shear stress and strain is nonlinear, which means the effective viscosity is not a constant. This relationship may be modeled by many numerical models including the Cross model [14], the Bingham-plastic model [18-20], and the power-law model [17]. In the current study, the Cross model is used, which can be expressed as follows [14]:

$$
\mu_{\mathrm{eff}}=\frac{\mu_{0}+K \mu_{B} \dot{\gamma}}{1+K \dot{\gamma}}
$$

where $\mu_{B}$ is plastic viscosity, $\mu_{0}=1000 \mu_{B}, K=\mu_{0} / \tau_{B}$, and $\tau_{B}$ is yield stress. $\dot{\gamma}$ is the strain rate and generally defined as the second invariant of the shear strain as

$$
\dot{\gamma}=\sqrt{\frac{1}{2} \sum_{i} \sum_{j} \gamma_{i j} \gamma_{i j}}
$$

In $2 \mathrm{D}$ case,

$$
\dot{\gamma}=\sqrt{\frac{1}{2}\left(\left(\frac{\partial u}{\partial x}\right)^{2}+\left(\frac{\partial v}{\partial y}\right)^{2}+\frac{1}{2}\left(\frac{\partial u}{\partial y}+\frac{\partial v}{\partial x}\right)^{2}\right)} .
$$

2.2. The Solution Algorithm. The SCC flow is the incompressible flow and (1) and (2) can be solved by the explicit threestep projection scheme [17]. At the first step, (2) is solved by only considering the body force and neglecting other forces. An intermediate velocity is computed as

$$
\overrightarrow{\mathbf{u}}_{n+1}^{*}=\overrightarrow{\mathbf{u}}_{n}^{*}+\overrightarrow{\mathbf{g}} \Delta t
$$

where the subscript $n$ denotes the time step. At the second step, the computed intermediate velocity is used to compute the strain rate, the effective viscosity, and the divergence of the shear stress:

$$
\frac{1}{\rho} \nabla \cdot \overrightarrow{\boldsymbol{\tau}}=\overrightarrow{\mathbf{S}}
$$

At the end of the second step, the second intermediate velocity is computed by

$$
\overrightarrow{\mathbf{u}}_{n+1}^{* *}=\overrightarrow{\mathbf{u}}_{n+1}^{*}+\overrightarrow{\mathbf{S}} \Delta t=\overrightarrow{\mathbf{u}}_{n}^{*}+(\mathbf{g}+\overrightarrow{\mathbf{S}}) \Delta t .
$$

The position is updated as

$$
\overrightarrow{\mathbf{r}}_{n+1}^{* *}=\overrightarrow{\mathbf{r}}_{n}+\overrightarrow{\mathbf{u}}_{n+1}^{* *} \Delta t
$$

where $\overrightarrow{\mathbf{r}}=(x, y)$ is the position vector. The following pressure Poisson equation is solved to get the pressure, which is needed 
to enforce the incompressibility [21]:

$$
\nabla \cdot\left(\frac{1}{\rho} \nabla p\right)=\frac{\nabla \cdot \overrightarrow{\mathbf{u}}_{n+1}^{* *}}{\Delta t}
$$

At the third step, the computed pressure is added to (2) to get a divergence-free velocity field at time $n+1$ :

$$
\overrightarrow{\mathbf{u}}_{n+1}=\overrightarrow{\mathbf{u}}_{n+1}^{* *}-\left(\frac{1}{\rho} \nabla p\right) \Delta t
$$

The position at $n+1$ time step is computed as

$$
\overrightarrow{\mathbf{r}}_{n+1}=\overrightarrow{\mathbf{r}}_{n}+\frac{1}{2}\left(\overrightarrow{\mathbf{u}}_{n}+\overrightarrow{\mathbf{u}}_{n+1}\right) \Delta t
$$

2.3. The SPH Formulation. The $\mathrm{SPH}$ is an interpolation method, which transforms the Partial Difference Equation (PDE) to integration form and allows any function to be expressed in terms of the value at a set of disordered particles. Following Gingold and Monaghan [5], a function $f(\overrightarrow{\mathbf{r}})$ at location $\overrightarrow{\mathbf{r}}$ can be defined by

$$
f(\overrightarrow{\mathbf{r}})=\int_{\Omega} f\left(\overrightarrow{\mathbf{r}}^{\prime}\right) W\left(\left|\overrightarrow{\mathbf{r}}-\overrightarrow{\mathbf{r}}^{\prime}\right|, h\right) d \overrightarrow{\mathbf{r}}^{\prime},
$$

where the integration is computed over the entire domain $\Omega$, $\left|\overrightarrow{\mathbf{r}}-\overrightarrow{\mathbf{r}}^{\prime}\right|$ is the scalar distance between $\overrightarrow{\mathbf{r}}$ and $\overrightarrow{\mathbf{r}}^{\prime}, h$ is smoothing length, and $W$ is interpolation kernel or kernel (smoothing) function.

The SCC domain may be represented by discrete particles and (15) can in turn be converted to the discretized form using the properties of all the particles inside the support domain as

$$
f\left(\overrightarrow{\mathbf{r}}_{a}\right)=\sum_{b} f\left(\overrightarrow{\mathbf{r}}_{b}\right) W\left(r_{a b}, h\right) V_{b}=\sum_{b} f_{b} W_{a b} V_{b}
$$

where $b$ represents the particle inside the support domain, $f_{b}$ is the value of function $f(\overrightarrow{\mathbf{r}})$ at location $\overrightarrow{\mathbf{r}}_{b}, r_{a b}=\left|\overrightarrow{\mathbf{r}}_{a b}\right|=\mid \overrightarrow{\mathbf{r}}_{a}-$ $\overrightarrow{\mathbf{r}}_{b} \mid$ is the distance between particle $a$ and $b, W_{a b}$ is the kernel value or contribution, and $V_{b}$ is the volume of particle $b$.

In the current study, the quintic kernel function [22] is used and can be expressed as

$$
W(q)=\alpha_{D}\left(1-\frac{q}{2}\right)^{4}(2 q+1) \quad 0 \leq q \leq 2,
$$

where $\alpha_{D}=7 /\left(4 \pi h^{2}\right), q=r_{a b} / h$, and $h$ is the smoothing length. The order of quintic kernel function is higher than the widely used cubic spline kernel function [14, 17, 23]. Hence, it has smoother kernel value and the first derivate, which is important for kernel interpolation and derivate computation.

Many forms have been proposed to evaluate the gradient and divergence in the SPH literatures. In the current study, the velocity divergence is computed by the antisymmetric form to improve the accuracy $[23,24]$ :

$$
\nabla \cdot \overrightarrow{\mathbf{u}}_{a}=\sum_{b=1}^{N}\left(\overrightarrow{\mathbf{u}}_{b}-\overrightarrow{\mathbf{u}}_{a}\right) \cdot \overrightarrow{\nabla_{a} W_{a b}} V_{b}
$$

where $\overrightarrow{\nabla_{a} W_{a b}}=\left(\left(\overrightarrow{\mathbf{r}}_{a}-\overrightarrow{\mathbf{r}}_{b}\right) / r_{a b}\right)\left(\partial W_{a b} / \partial r_{a b}\right)=-\overrightarrow{\nabla_{b} W_{a b}}$ is the kernel gradient. The pressure gradient is evaluated by the symmetric form to conserve the linear and angular momentum $[14,17,18]$ :

$$
\nabla p_{a}=\rho_{a} \sum_{b=1}^{N} m_{b}\left(\frac{p_{a}}{\rho_{a}^{2}}+\frac{p_{b}}{\rho_{b}^{2}}\right) \overrightarrow{\nabla_{a} W_{a b}}
$$

The Laplacian operator in the pressure Poisson equation is formulated as a hybrid of a standard SPH first derivative with a finite difference approximation for the first derivative [17, 20]:

$$
\nabla \cdot\left(\frac{1}{\rho} \nabla p\right)=\sum_{b} m_{b} \frac{8}{\left(\rho_{a}+\rho_{b}\right)} \frac{P_{a b} \overrightarrow{\mathbf{r}}_{a b}}{r_{a b}{ }^{2}+\eta^{2}} \cdot \overrightarrow{\nabla W_{a b}} V_{b},
$$

where $P_{a b}=P_{a}-P_{b}$ and $\eta=0.01 h$ is a small number introduced to keep the denominator no-zero.

The gradient of shear stress is computed similar to the pressure gradient and can be expressed by

$$
\frac{1}{\rho} \nabla \cdot \overrightarrow{\boldsymbol{\tau}}=\nabla m_{b}\left(\frac{\overrightarrow{\boldsymbol{\tau}}_{a}}{\rho_{a}^{2}}+\frac{\overrightarrow{\boldsymbol{\tau}}_{b}}{\rho_{b}^{2}}\right) \cdot \overrightarrow{\nabla W_{a b}}
$$

The smoothing length is an important parameter for SPH simulation, which influences the accuracy of SPH interpolation. For particles near the free surface, which has less neighboring particles, due to the absence of particles above the free surface, the number of neighboring particles is less than the inner particles. This may cause the accuracy of SPH interpolation to be reduced. In this paper, the smoothing length of the particle changes depended on the neighboring particle number (e.g., $n_{\text {min }}=40$ ) and the smoothing length expands with the following scheme for each step:

(1) The particle assigns the initial smoothing length $h=2 \Delta$, where $\Delta$ is the particle size. The particle computes the number of neighboring particles $(N)$ using smoothing length $h$.

(2) If $N>n_{\text {min }}$, the particle's smoothing length for current step is $h=2 \Delta$.

(3) If $N<n_{\min }$, the particle increases the smoothing with $h_{\text {new }}=h+\Delta h=h+\Delta / 100$ and computes the number of neighboring particles $N$.

(4) The particle will continue to increase the smoothing length until the smoothing length becomes larger than $3 \Delta$ or the number of neighboring particles becomes larger than $N$.

The abovementioned algorithm has two advantages. The first is that the particles, especially those near the free surface, have more neighboring particles, and the accuracy of the 


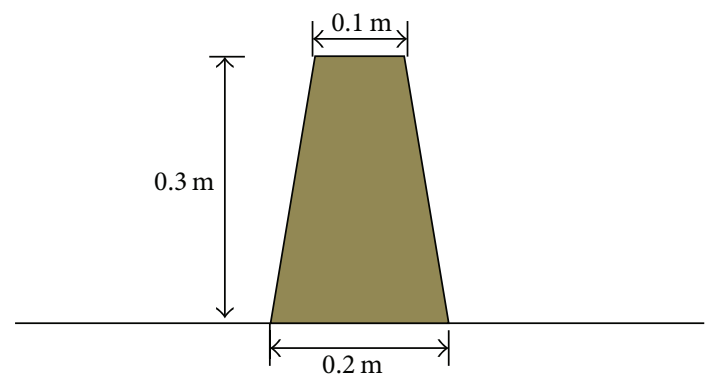

FIGURE 1: The initial configuration of the placement for slump flow.

kernel interpolation will be increased. The second is that the smoothing length is linearly distributed and only 101 smoothing lengths were used. It is easy to construct the kernel table and each particle saves the index of the kernel table by the smoothing length. In the simulation, the kernel properties $\left(\alpha_{D}\right.$ in (17)) can be easily gotten by indexing the smoothing table.

2.4. The Boundary Condition. There are several methods to model the boundary condition including the virtual force method [14], the fixed wall particle method $[9,14,17]$, and the mirror particle method [21, 24-26]. Compared with the virtual force method, the mirror particle method can predict accurate pressure near the wall boundary and it can further reduce the pressure oscillation [25]. Compared with the fixed wall particle method, the mirror particles are mirrored from the fluid particles instead of solving the NS equations. Hence, the number of particles in the NS equation is less than the fixed wall particle method and can save the simulation time. By considering the advantages of mirror particle method, it is adopted in current study to treat the wall boundary. The position of the mirror particles is computed by mirroring the SCC particles. The volume, density, and effective viscosity are set equal to their reference SCC particles. The velocity of the mirror particle is set to zero to simulate the partial slip of wall boundary condition. The pressure of the mirror particle is computed by $p_{m}=p_{s}+\rho_{s} \overrightarrow{\mathbf{r}}_{m s} \cdot \overrightarrow{\mathbf{g}}$, where the subscripts $m$ and $s$ denote the mirror and SCC particle, respectively.

\section{Applications in SCC Modeling}

In this section, the improved SPH model is applied to a benchmark slump test model to simulate the slum flow of SCC to demonstrate the capability of the proposed model in SCC flow modeling. And the model is also used to simulate the filling ability of SCC by using the L-box test.

3.1. Slump Test. Figure 1 illustrates the configuration of the slump cone which is used for slump testing according to BS EN 12350-8. Numerical simulation of the SCC flow is carried out in two-dimensional configurations.

The SCC is a high performance mix and the density and rheological properties of SCC used for slump test are presented in Table 1 [16]. The mixture has a plastic viscosity
TABLE 1: Density and rheological properties of SCC for simulation [16].

\begin{tabular}{cccc}
\hline & Density $\left(\mathrm{kg} / \mathrm{m}^{3}\right)$ & Plastic viscosity $(\mathrm{Pa} \mathrm{s})$ & Yield stress $(\mathrm{Pa})$ \\
\hline SCC & 2380 & 48.4 & 56.3 \\
\hline
\end{tabular}

at $4 \mathrm{~Pa} \cdot \mathrm{s}$ and yield stress at $200 \mathrm{~Pa}$ with the density at $2380 \mathrm{~kg} / \mathrm{m}^{3}$.

The simulation domain is discretized by particles with size $\Delta=2 \mathrm{~mm}$; in total, 11268 particles are involved in the simulation. The particle size was selected after the convergence study was done. The time step size is dynamically adjusted according to the CFL number (equal to 0.2 in this study). The slump cone movement is important for the simulation results. In order to keep the consistency with BS EN 12350-8 standard and considering the numerical stability, the slump cone is initially located $0.01 \mathrm{~m}$ above the bottom. As the simulation starts, the slump cone is fixed at the first $0.1 \mathrm{~s}$. At $t=0.1 \mathrm{~s}$, it starts to move upward with fixed speed of $0.1 \mathrm{~m} / \mathrm{s}$ until the simulation finishes. It is important for the slump cone to keep the $0.01 \mathrm{~m}$ distance from the bottom. If the slump cone put on the bottom, there will be no free surface in the domain and the Poisson equation is difficult to solve. The slump cone starts to move at $0.1 \mathrm{~s}$ as assumed. That means the slump cone initially located at the bottom and in $0.1 \mathrm{~s}$ it moves $0.01 \mathrm{~m}$ to compensate the difference with the standard.

Figures 2-4 show the various stages of the $2 \mathrm{D}$ flow profile during the numerical simulations of slump test of SCC including the contour of the distribution of the velocity, pressure, and the shear strain rate, respectively. It is evident from the results that the improved SPH method is able to characterize the flow of SCC at different stages. It should be also noted that the initial profiles of the free surfaces are affected by the motion of the slump cone with its fast removal. The available experimental test data from literature [21] is used for comparison. The model is capable of capturing all the key characteristics of the SCC flow including the pressure, velocity, shear strain rate, and viscosity at different locations. However, only the spread value is available for comparison. By comparing the spread value from the simulation results with the test value, the simulation provides reasonable agreement with the experimental results with less than $20 \%$ of discrepancies.

3.2. L-Box Test. In order to understand the filling ability in addition to the flow characteristics of SCC, the classic Lbox filling test is also simulated by using the improved $\mathrm{SPH}$ method. Figure 5 illustrates the configuration of the physical L-box test and its dimension. The apparatus consists of rectangular section box in the shape of an "L," with a vertical and horizontal section, separated by a movable gate, in front of which vertical lengths of reinforcement bar are fitted. The vertical section is filled with concrete, and then the gate was lifted to let the concrete flow into the horizontal section. This test is to measure the filling and passing ability of concrete to flow through tight obstructions without segregation or blocking. 


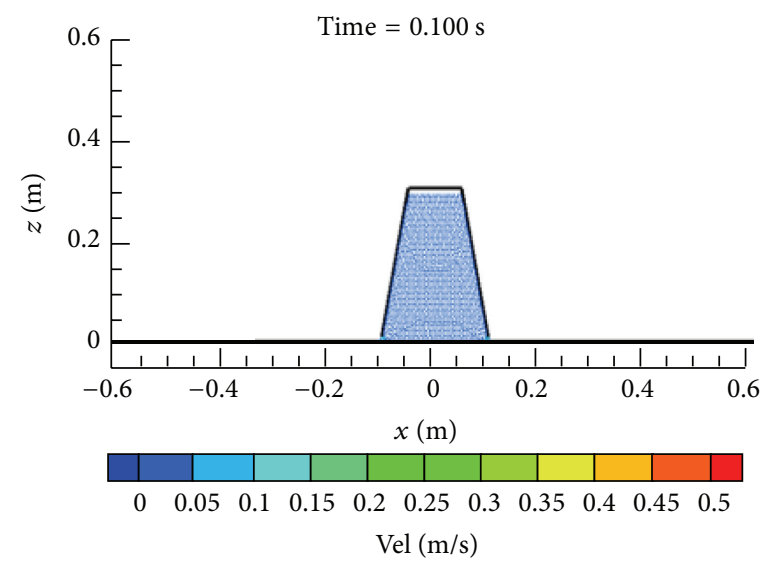

(a) $t=0.1 \mathrm{sec}$
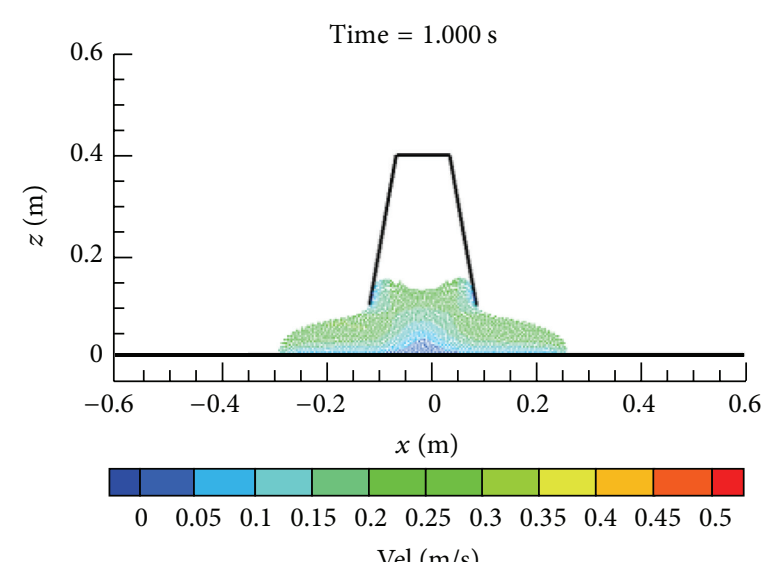

(c) $t=1.0 \mathrm{sec}$

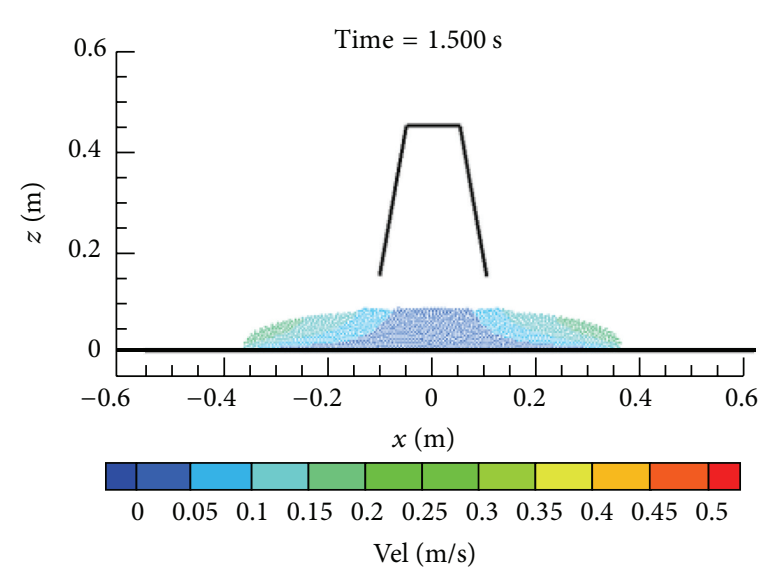

(e) $t=1.5 \mathrm{sec}$

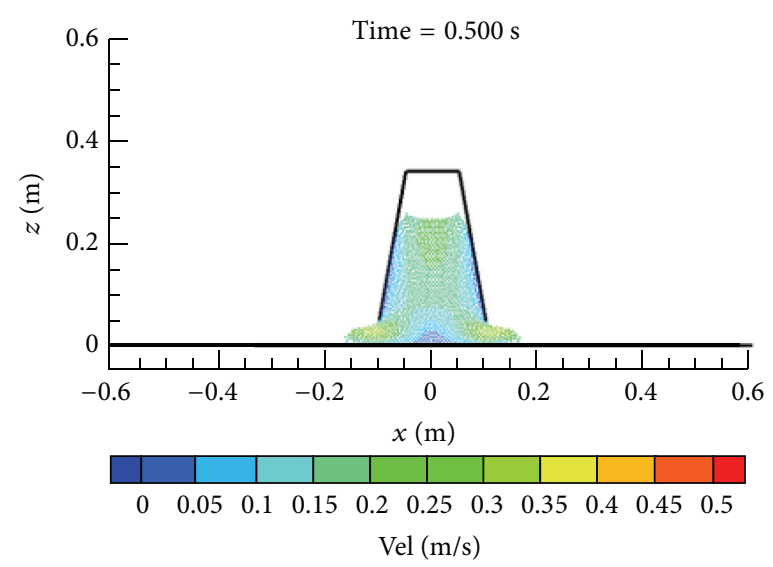

(b) $t=0.5 \mathrm{sec}$

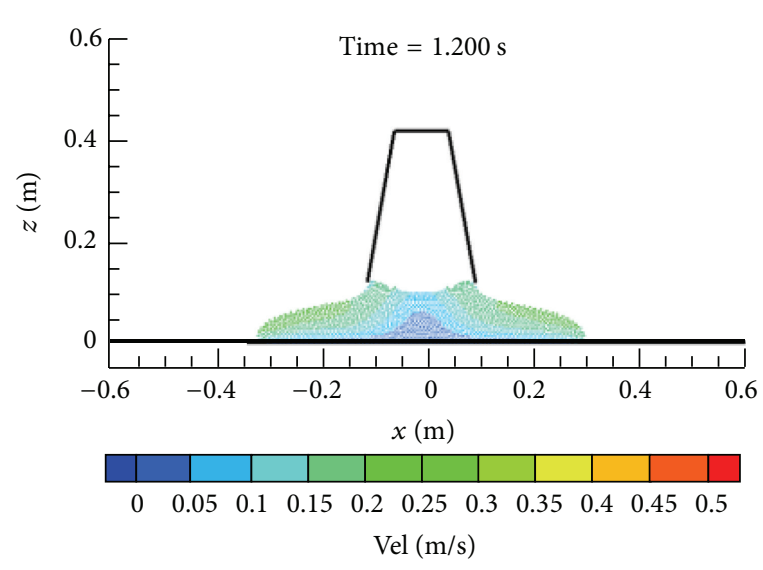

(d) $t=1.2 \mathrm{sec}$

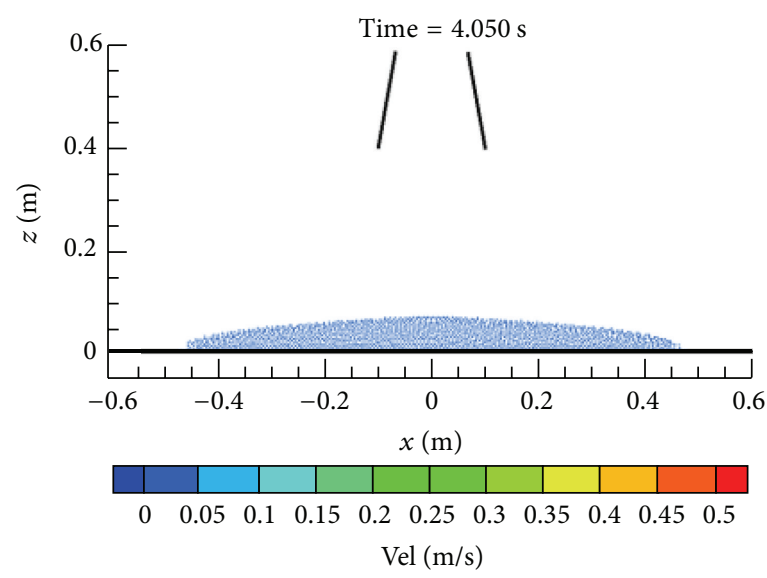

(f) Final spread

FIGURE 2: The velocity distribution during slump flow.

The same SCC mix properties as presented in Table 1 are used for L-box model. The mixture has a plastic viscosity at $4 \mathrm{~Pa} \cdot \mathrm{s}$ and yield stress at $200 \mathrm{~Pa}$ with the density at $2380 \mathrm{~kg} / \mathrm{m}^{3}$. Figures $6-8$ present the distribution of velocity, pressure, and strain rate during the flow of SCC in an L-box, at $0.2 \mathrm{~s}, 0.6 \mathrm{~s}, 1.5 \mathrm{~s}$, and $10 \mathrm{~s}$, respectively. It can be observed that, due to the friction between the SCC and the wall, the particles very close to the wall surfaces take longer time to flow. The flowing and filling ability of the SCC can be well captured by this model.

The simulation domain is discretized by particles with size $\Delta=2 \mathrm{~mm}$; in total, 18000 particles are involved in 


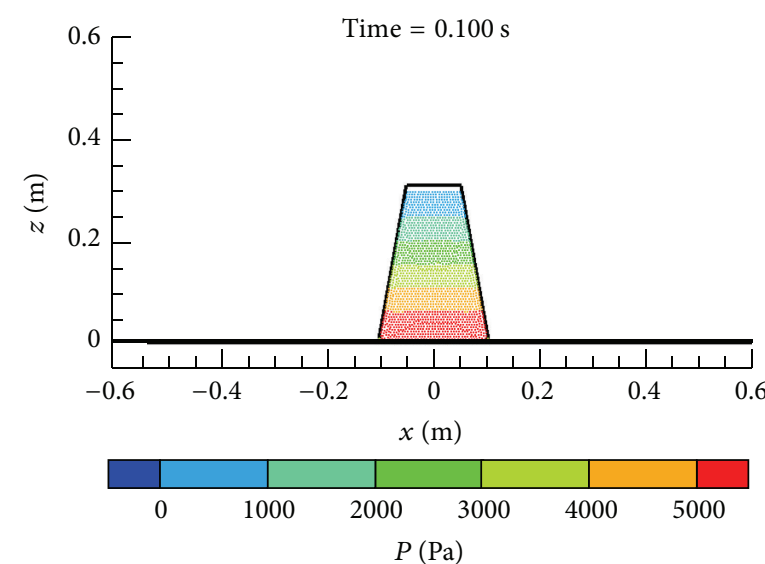

(a) $t=0.1 \mathrm{sec}$

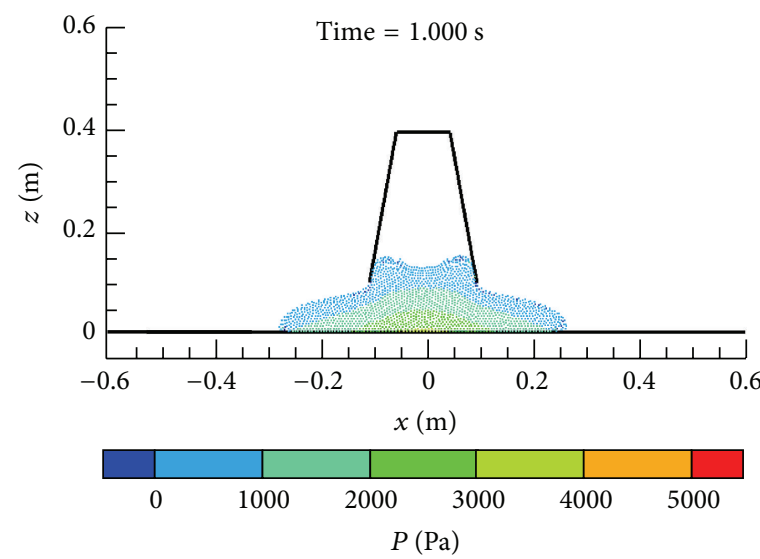

(c) $t=1.0 \mathrm{sec}$

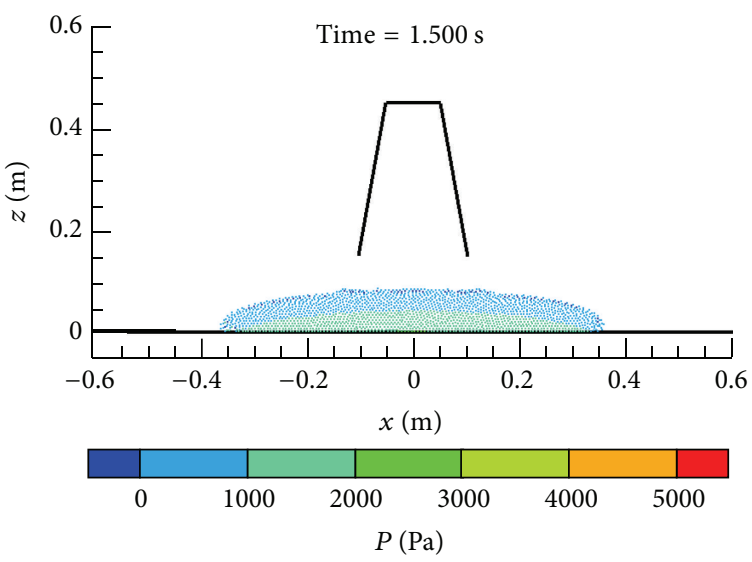

(e) $t=1.5 \mathrm{sec}$

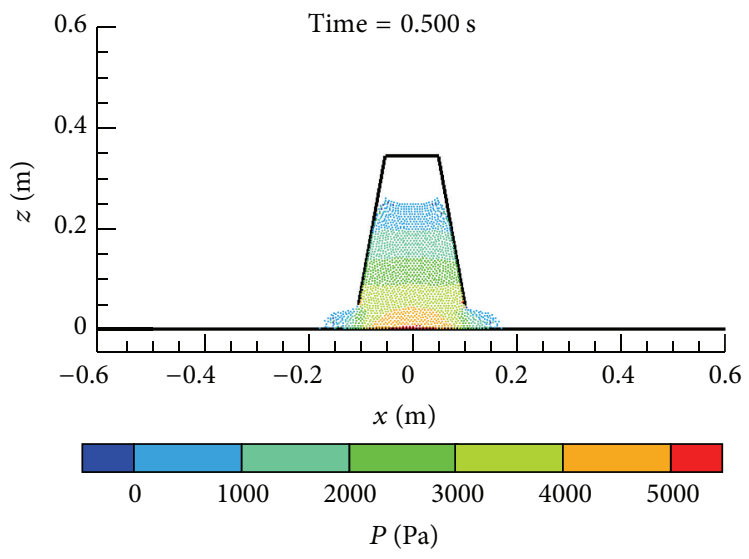

(b) $t=0.5 \mathrm{sec}$

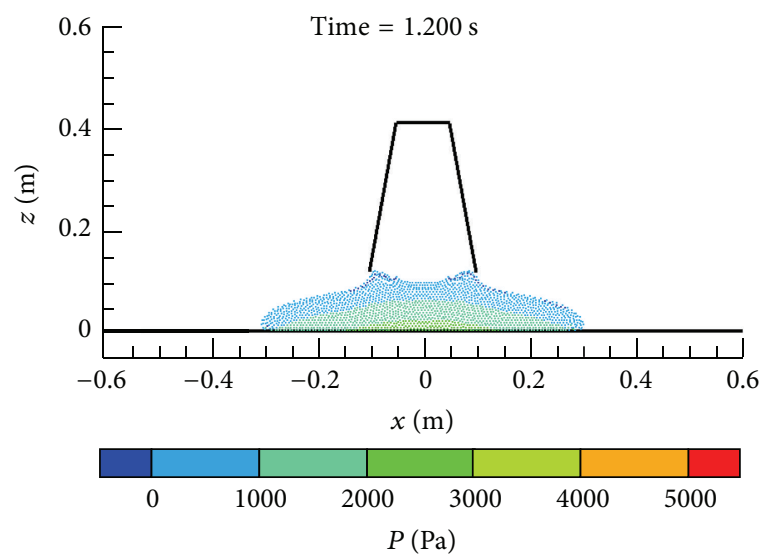

(d) $t=1.2 \mathrm{sec}$

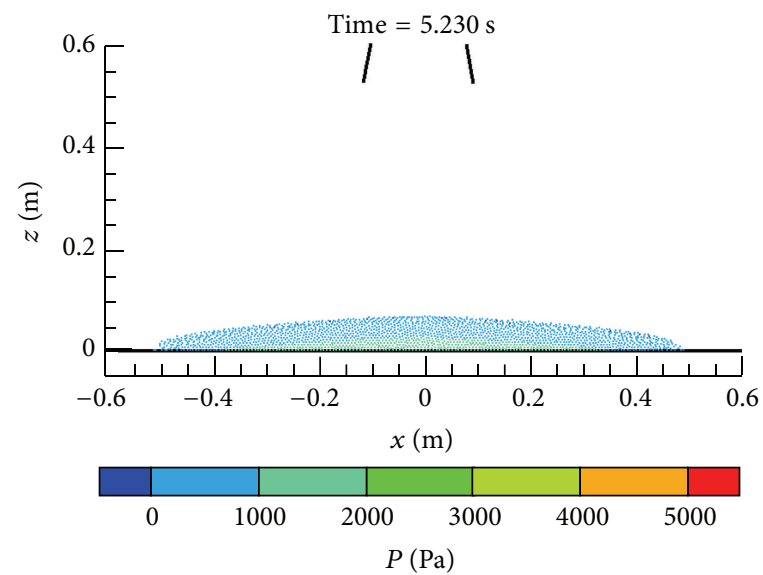

(f) Final spread

FIgURE 3: The pressure distribution during slump flow.

the simulation. The particle size was selected after the convergence study was done. The time step size is dynamically adjusted according to the CFL number (equal to 0.2 in this study).

\section{Conclusions}

Due to the large deformation at the flowing process of SCC, an enhanced Lagrangian particle-based method-an improved SPH method-with its exceptional advantages 

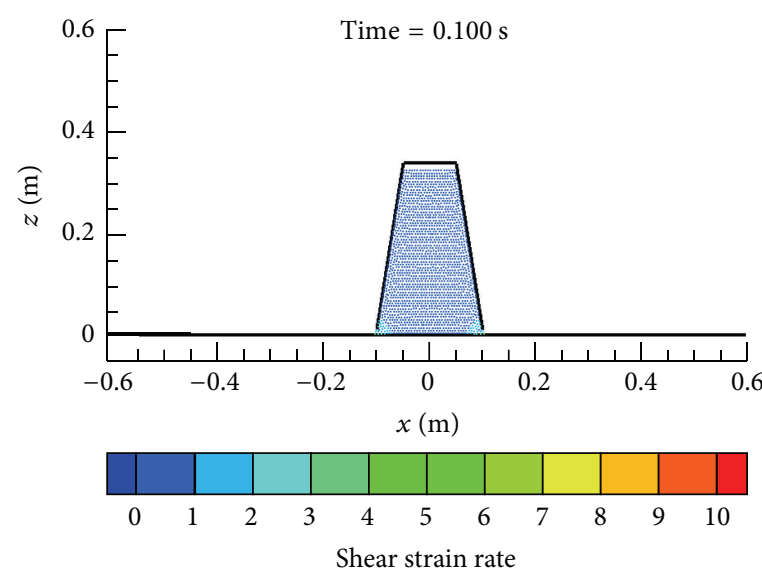

(a) $t=0.1 \mathrm{sec}$
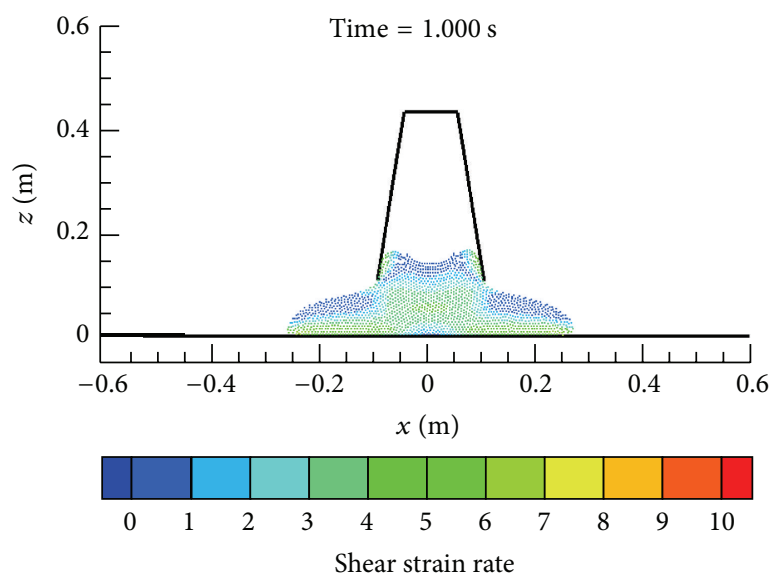

(c) $t=1.0 \mathrm{sec}$
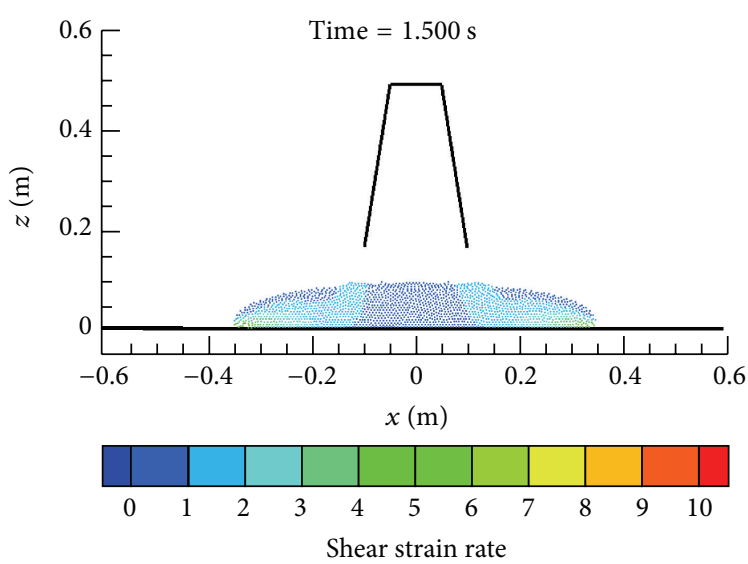

(e) $t=1.5 \mathrm{sec}$

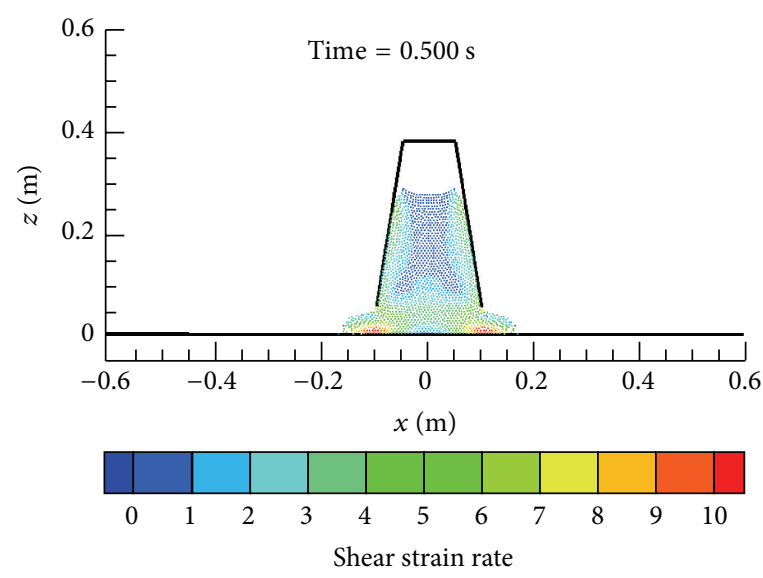

(b) $t=0.5 \mathrm{sec}$
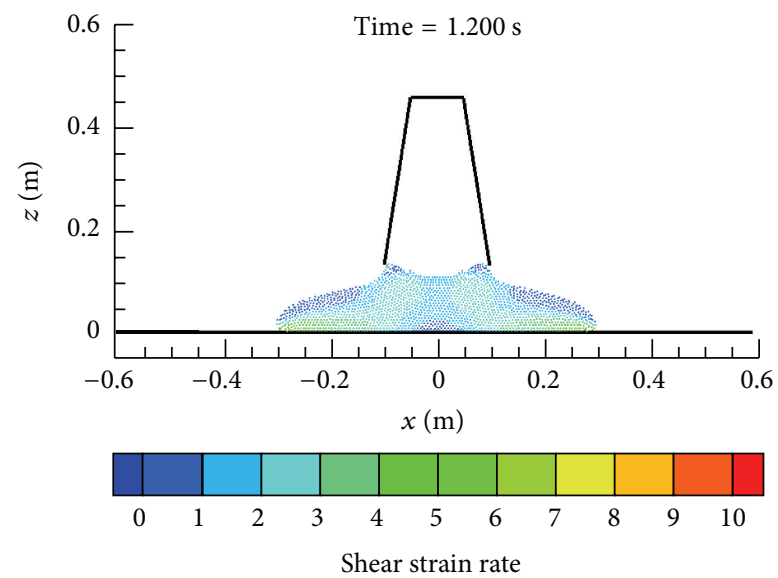

(d) $t=1.2 \mathrm{sec}$
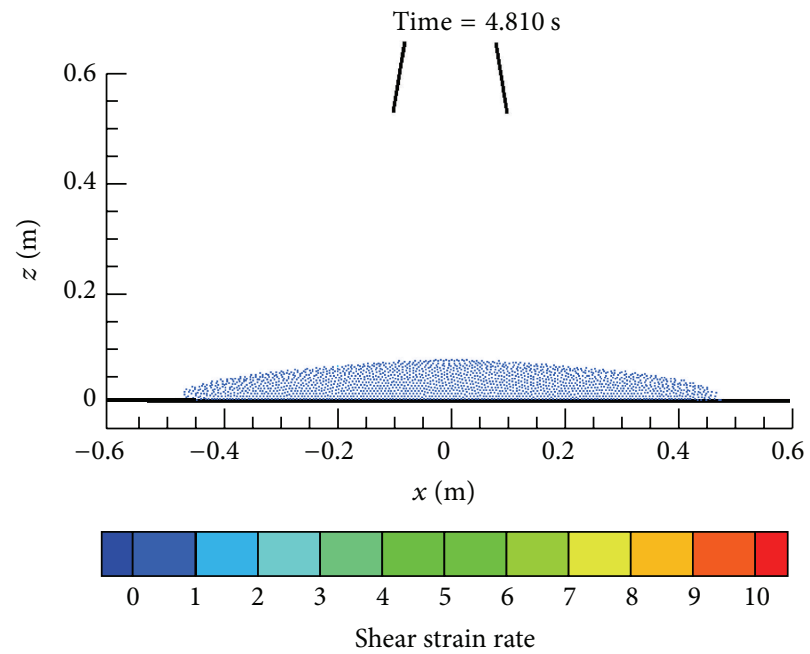

(f) Final spread

FIGURE 4: The shear strain rate distribution during slump flow. 


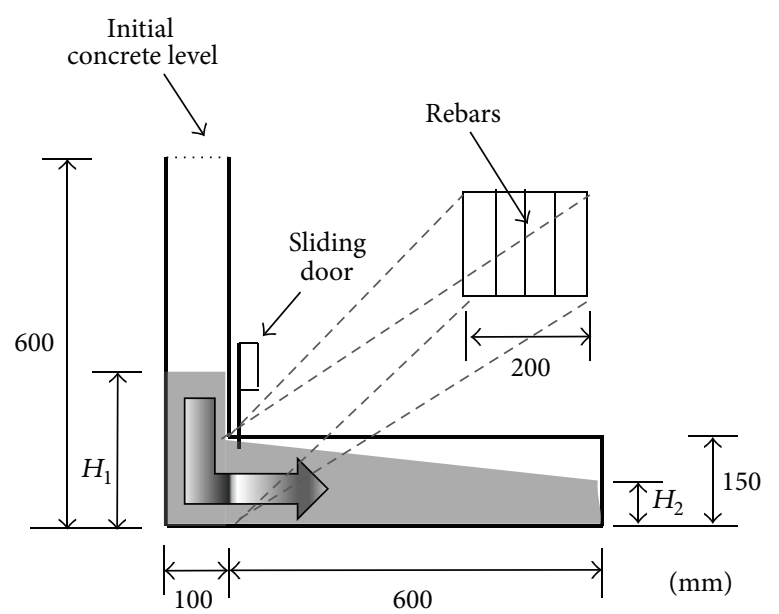

FIgURE 5: The configuration of the L-box test for SCC [15].
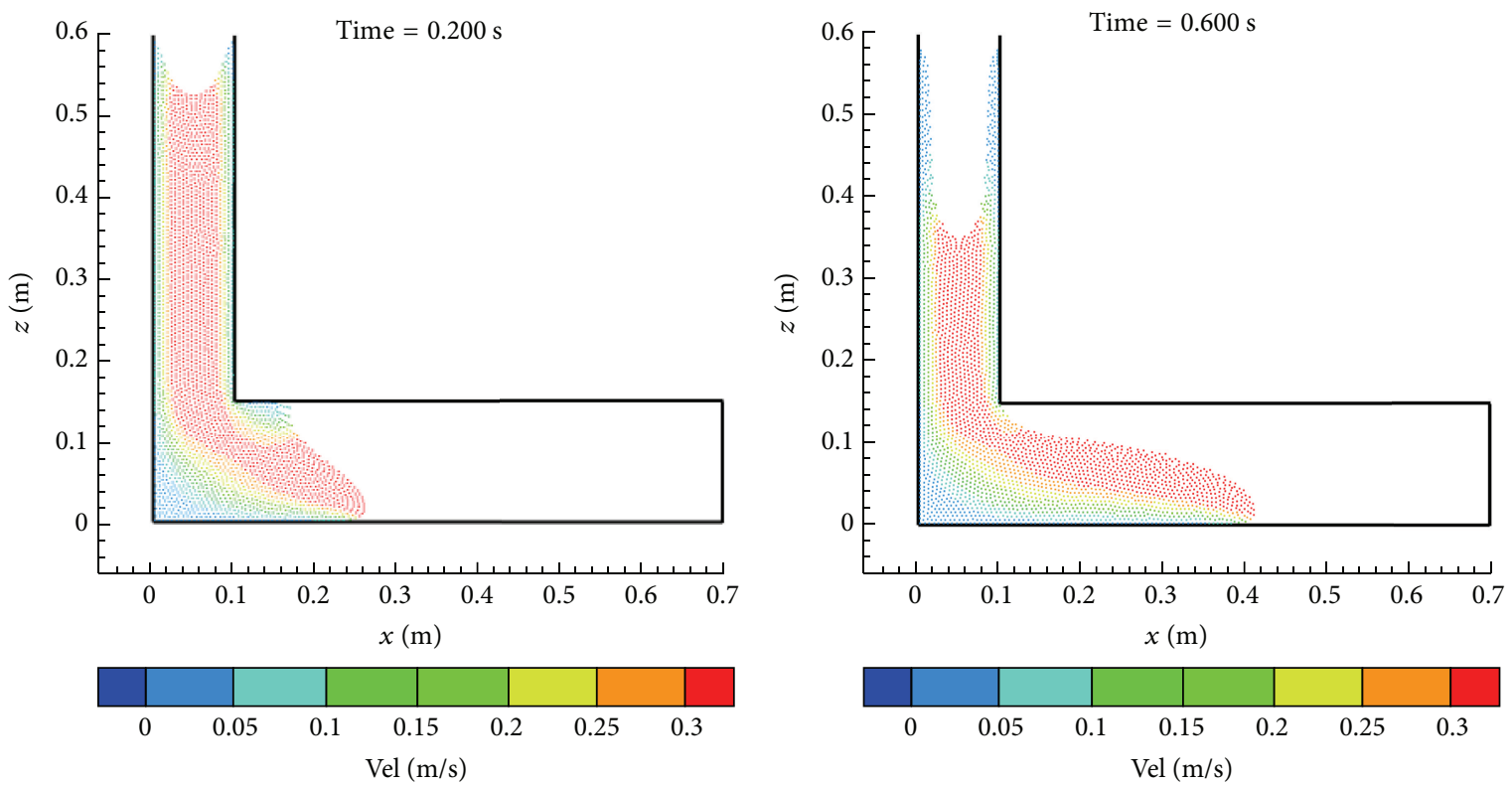

(a) $t=0.2 \mathrm{sec}$
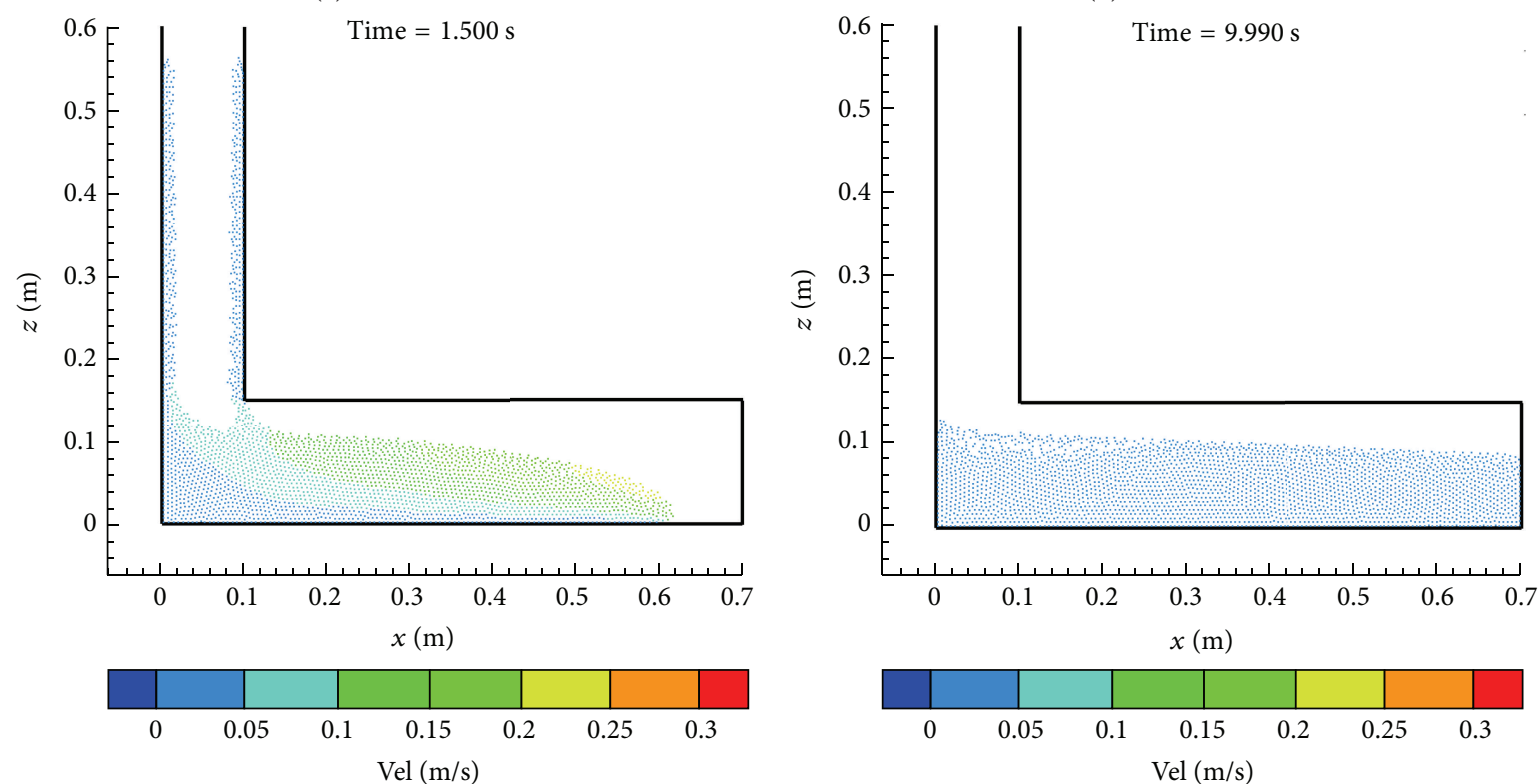

(d) $t=10 \mathrm{sec}$

(c) $t=1.5 \mathrm{sec}$

distribution. 


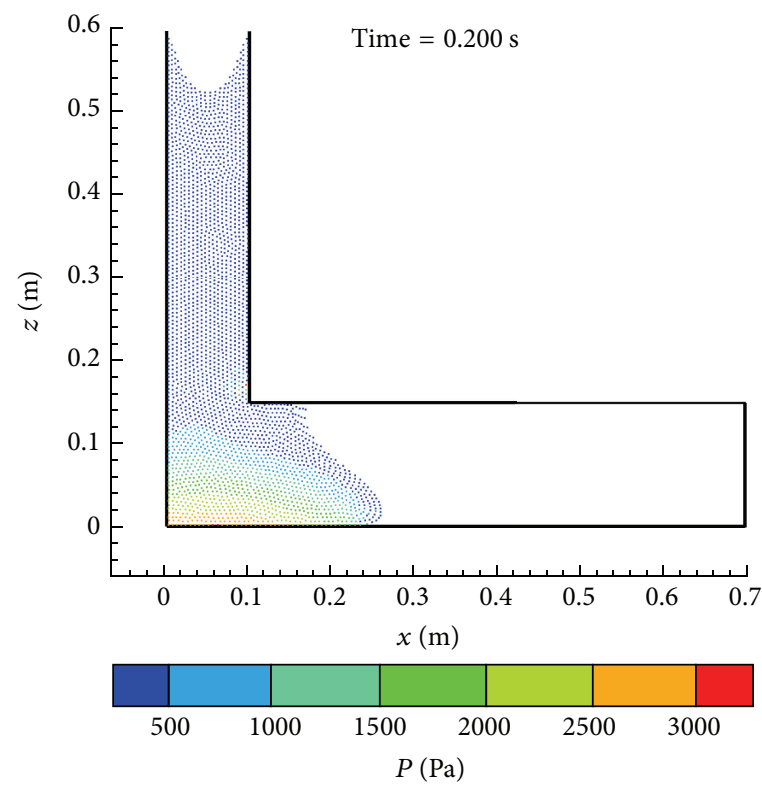

(a) $t=0.2 \mathrm{sec}$

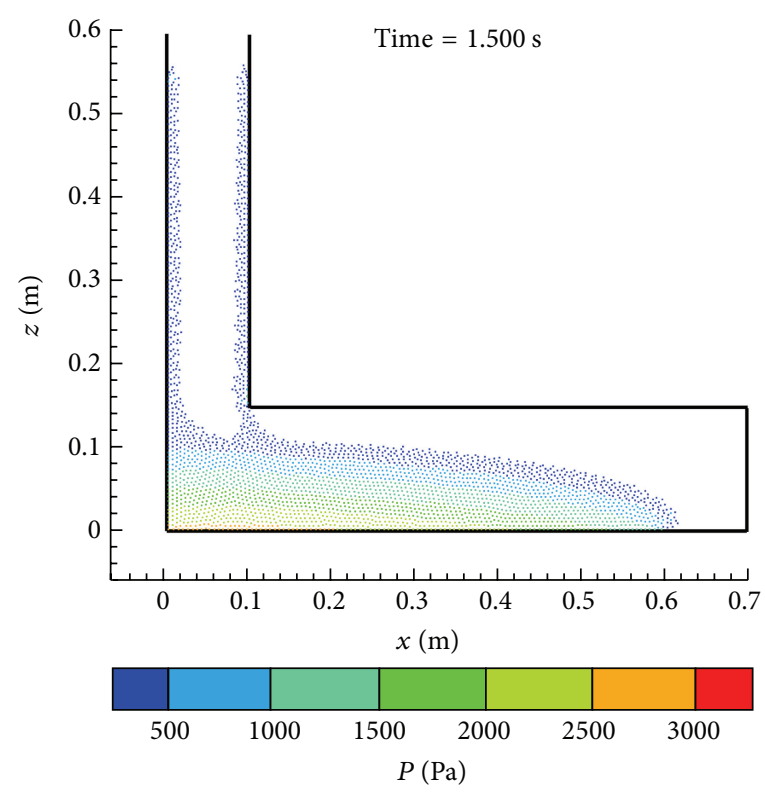

(c) $t=1.5 \mathrm{sec}$

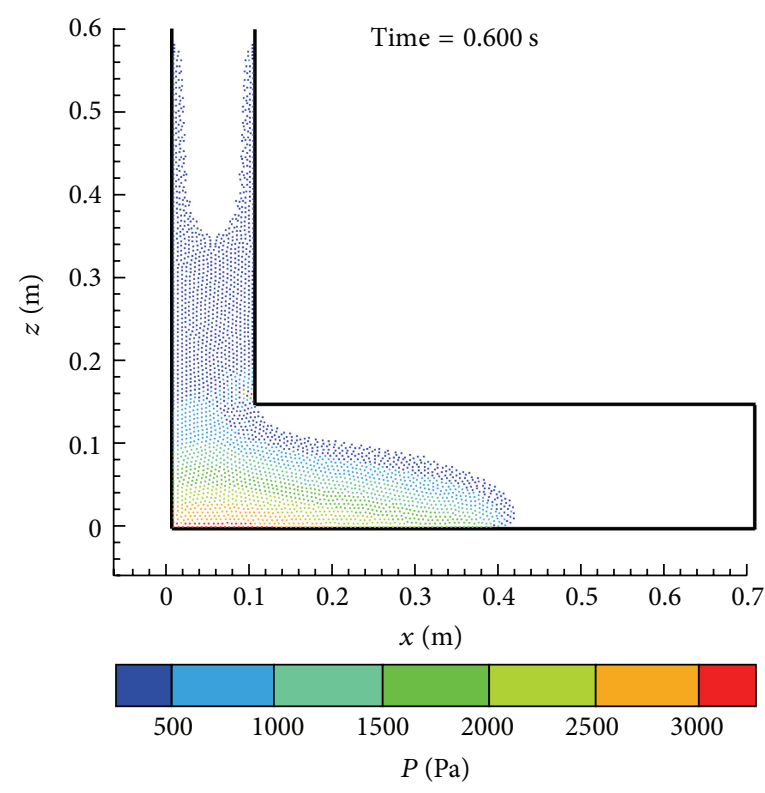

(b) $t=0.6 \mathrm{sec}$

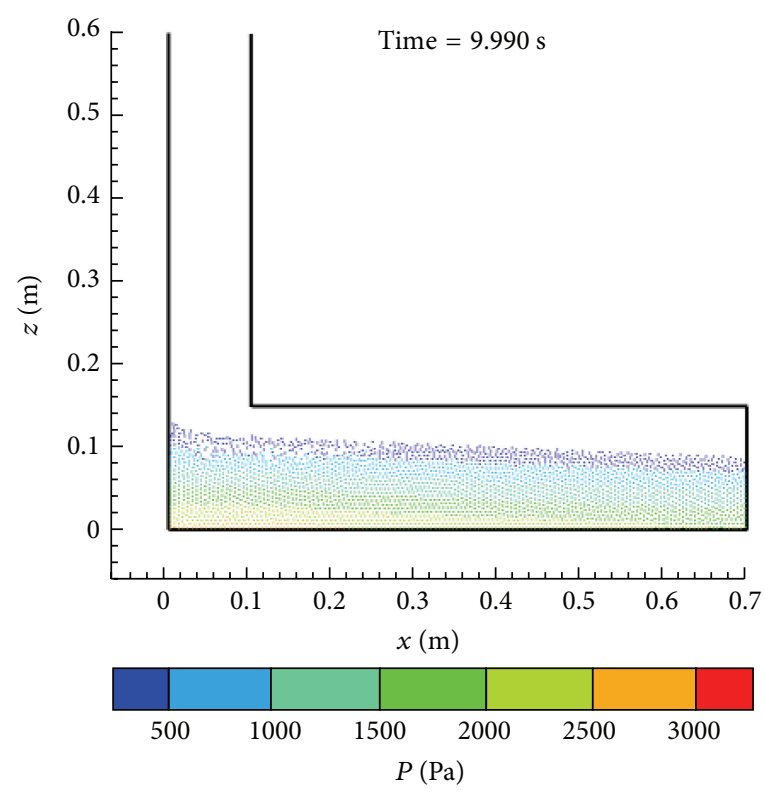

(d) $t=10 \mathrm{sec}$

FIGURE 7: Simulation of L-box testing for SCC with pressure distribution.

in solving problems involving fragmentation, coalescence, and violent free surface deformation is developed in this study to simulate the flow of SCC as a non-Newtonian fluid to achieve stable results with satisfactory convergence properties. Navier-Stokes equations and incompressible mass conservation equations are solved as basics. Cross rheological model is used to simulate the shear stress and strain relationship of SCC. Mirror particle method is used for wall boundaries. The improved SPH method is tested by a typical 2D slump flow problem and also applied to L-box flowing problem. The results obtained from this method are reasonably agreeing with the experimental data. This enables the modeling of the flow of SCC by enhancing the current particle method and also provides an efficient tool to estimate the lateral pressure of the SCC exerted on structures. Moreover, it will benefit the industry and practical concrete applications by using this method to estimate the flowability, the mould filling performance of concrete, and also the interactions between the concrete and mould during casting. The proposed method could also become an efficient method to study the mix design to optimize the workability of the concrete. 


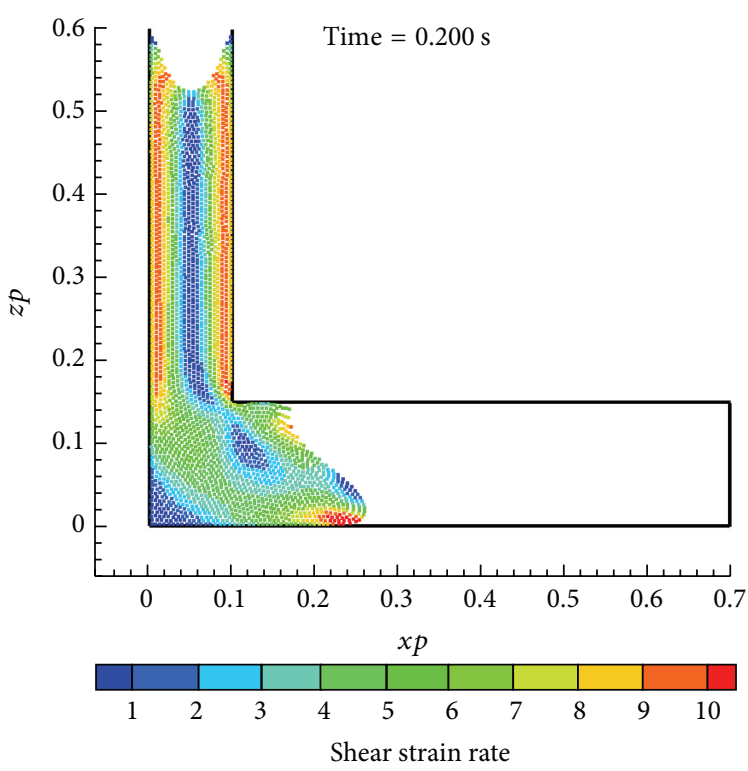

(a) $t=0.2 \mathrm{sec}$

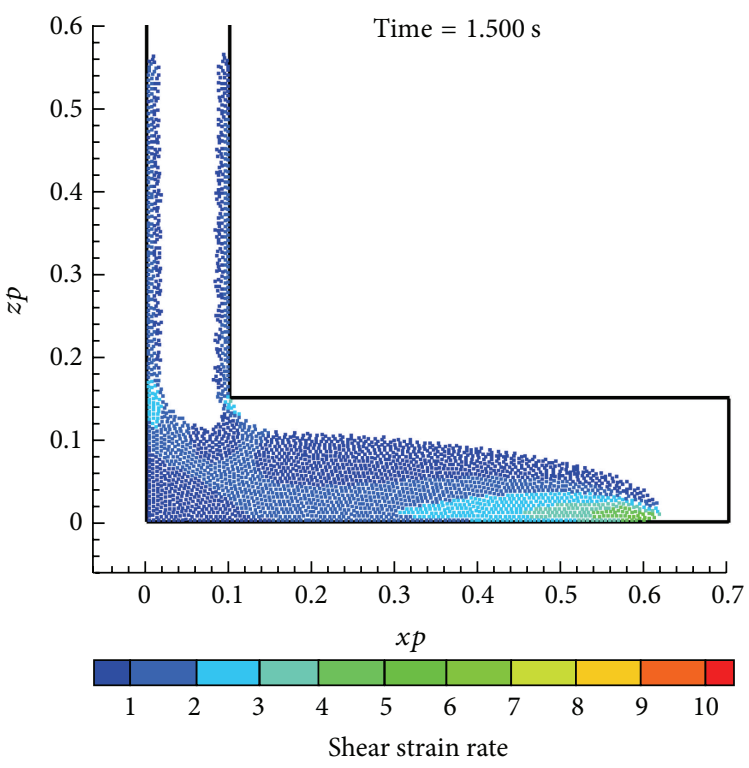

(c) $t=1.5 \mathrm{sec}$

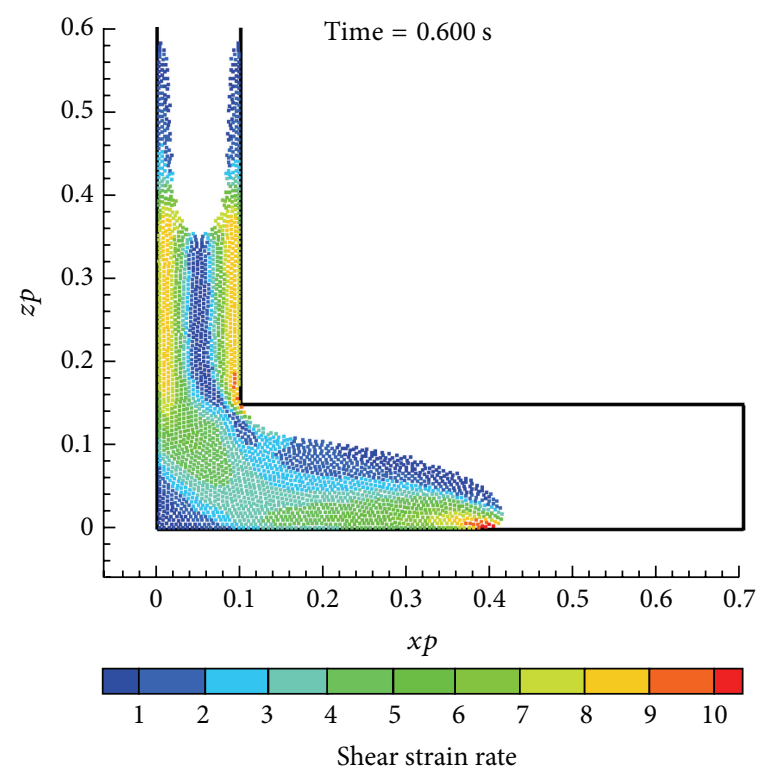

(b) $t=0.6 \mathrm{sec}$

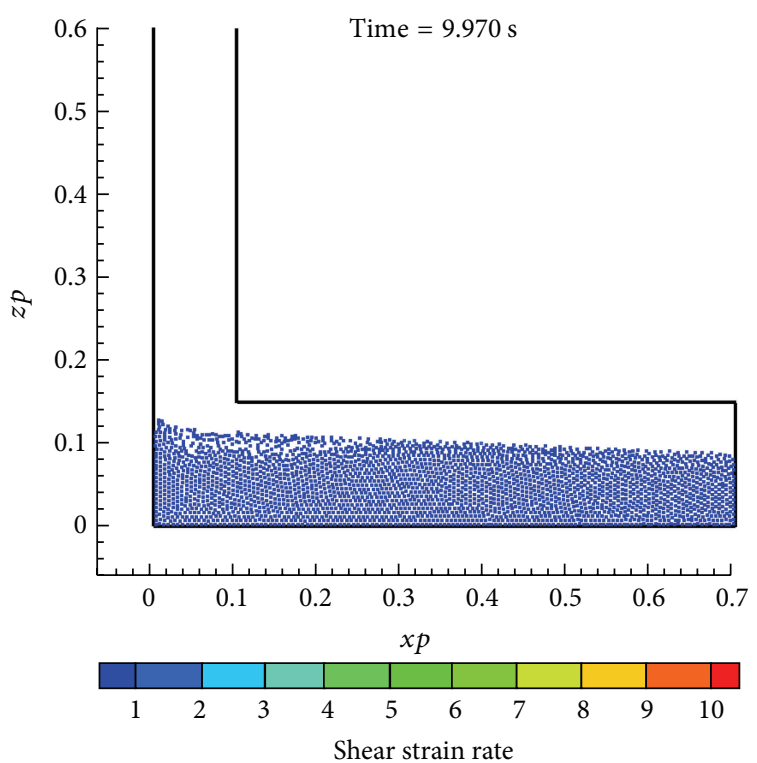

(d) $t=10 \mathrm{sec}$

FIGURE 8: Simulation of L-box testing for SCC with shear strain rate distribution.

\section{Competing Interests}

The authors declare that they have no competing interests.

\section{Acknowledgments}

Grateful acknowledgment is made to ECARD Research Grant scheme from Queensland University of Technology and the National Natural Science Foundation of China (Project no. 51508294) to collectively support this project.

\section{References}

[1] K. H. Khayat, "Workability, testing, and performance of selfconsolidating concrete," ACI Materials Journal, vol. 96, no. 3, pp. 346-353, 1999.
[2] J. E. Wallevik, "Rheological properties of cement paste: thixotropic behavior and structural breakdown," Cement and Concrete Research, vol. 39, no. 1, pp. 14-29, 2009.

[3] A. Ghanbari and B. L. Karihaloo, "Prediction of the plastic viscosity of self-compacting steel fibre reinforced concrete," Cement and Concrete Research, vol. 39, no. 12, pp. 1209-1216, 2009.

[4] N. Roussel, G. Ovarlez, S. Garrault, and C. Brumaud, "The origins of thixotropy of fresh cement pastes," Cement and Concrete Research, vol. 42, no. 1, pp. 148-157, 2012.

[5] R. A. Gingold and J. J. Monaghan, "Smoothed particle hydrodynamics: theory and application to non-spherical stars," Monthly Notices of the Royal Astronomical Society, vol. 181, no. 3, pp. 375389, 1977. 
[6] L. B. Lucy, "A numerical approach to the testing of the fission hypothesis," The Astronomical Journal, vol. 82, no. 12, pp. 10131024, 1977.

[7] J. J. Monaghan, "On the problem of penetration in particle methods," Journal of Computational Physics, vol. 82, no. 1, pp. $1-15,1989$.

[8] A. Colagrossi and M. Landrini, "Numerical simulation of interfacial flows by smoothed particle hydrodynamics," Journal of Computational Physics, vol. 191, no. 2, pp. 448-475, 2003.

[9] $\mathrm{H}$. Xu, Numerical simulation of breaking wave impact on structures [Ph.D. thesis], National University of Singapore, Singapore, 2013.

[10] J. Bonet and S. Kulasegaram, "Correction and stabilization of smooth particle hydrodynamics methods with applications in metal forming simulations," International Journal for Numerical Methods in Engineering, vol. 47, no. 6, pp. 1189-1214, 2000.

[11] Y.-P. Chui and P.-A. Heng, "A meshless rheological model for blood-vessel interaction in endovascular simulation," Progress in Biophysics \& Molecular Biology, vol. 103, no. 2-3, pp. 252-261, 2010.

[12] X. Xu, J. Ouyang, B. Yang, and Z. Liu, "SPH simulations of threedimensional non-Newtonian free surface flows," Computer Methods in Applied Mechanics and Engineering, vol. 256, pp. 101116, 2013.

[13] G. Zhou, W. Ge, and J. Li, "Smoothed particles as a nonNewtonian fluid: a case study in Couette flow," Chemical Engineering Science, vol. 65, no. 6, pp. 2258-2262, 2010.

[14] S. Shao and E. Y. M. Lo, "Incompressible SPH method for simulating Newtonian and non-Newtonian flows with a free surface," Advances in Water Resources, vol. 26, no. 7, pp. 787800, 2003.

[15] F. Carl, Curling and strain monitoring of slabs strips in a laboratory environment [M.S. thesis], Université Laval, Quebec City, Canada, 2005.

[16] F. Mukhtar, Computer-aided modelling and simulation of flow of self-compacting concrete [M.S. thesis], King Fahd University of Petroleum and Minerials, Dhahran, Sudi Arabia, 2011.

[17] S. M. Hosseini, M. T. Manzari, and S. K. Hannani, "A fully explicit three-step SPH algorithm for simulation of nonNewtonian fluid flow," International Journal of Numerical Methods for Heat \& Fluid Flow, vol. 17, no. 7-8, pp. 715-735, 2007.

[18] S. Kulasegaram, B. L. Karihaloo, and A. Ghanbari, "Modelling the flow of self-compacting concrete," International Journal for Numerical and Analytical Methods in Geomechanics, vol. 35, no. 6, pp. 713-723, 2011.

[19] H. Lashkarbolouk, A. M. Halabian, and M. R. Chamani, "Simulation of concrete flow in V-funnel test and the proper range of viscosity and yield stress for SCC," Materials and Structures, vol. 47, no. 10, pp. 1729-1743, 2014.

[20] H. Zhu, N. S. Martys, C. Ferraris, and D. D. Kee, "A numerical study of the flow of Bingham-like fluids in two-dimensional vane and cylinder rheometers using a smoothed particle hydrodynamics (SPH) based method," Journal of Non-Newtonian Fluid Mechanics, vol. 165, no. 7-8, pp. 362-375, 2010.

[21] S. J. Cummins and M. Rudman, "An SPH projection method," Journal of Computational Physics, vol. 152, no. 2, pp. 584-607, 1999.

[22] M. B. Liu and G. R. Liu, "Smoothed particle hydrodynamics (SPH): an overview and recent developments," Archives of Computational Methods in Engineering, vol. 17, no. 1, pp. 25-76, 2010.
[23] J. J. Monaghan, "Simulating free surface flows with SPH," Journal of Computational Physics, vol. 110, no. 2, pp. 399-406, 1994.

[24] A. Colagrossi, A Meshless Lagrangian Method for Free-Surface and Interface Flows with Fragmentation, Università di Roma La Sapienza, Rome, Italy, 2003.

[25] X. Liu, H. Xu, S. Shao, and P. Lin, "An improved incompressible SPH model for simulation of wave-structure interaction," Computers \& Fluids, vol. 71, pp. 113-123, 2013.

[26] E. Y. M. Lo and S. Shao, "Simulation of near-shore solitary wave mechanics by an incompressible SPH method," Applied Ocean Research, vol. 24, no. 5, pp. 275-286, 2002. 

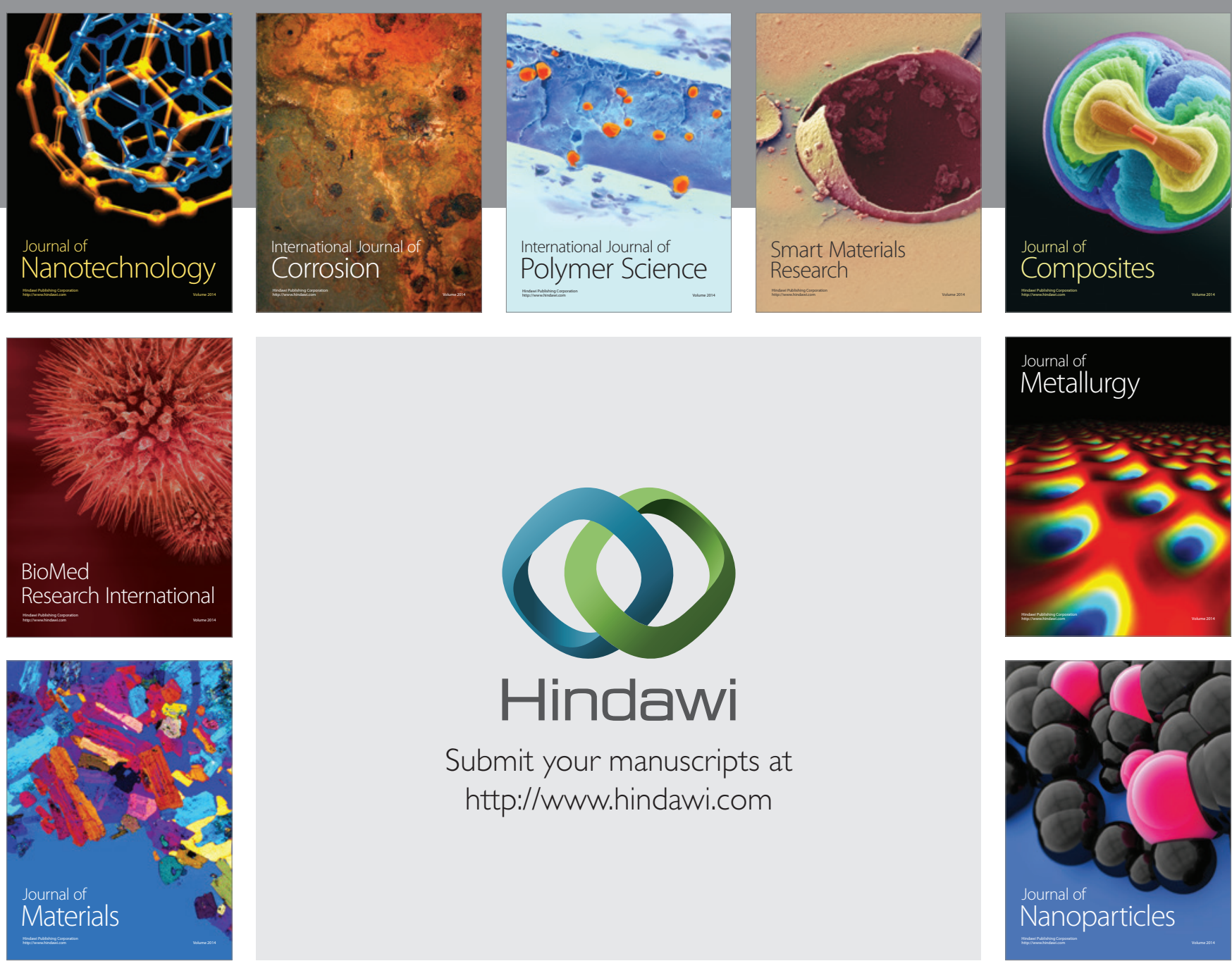

\section{Hindawi}

Submit your manuscripts at

http://www.hindawi.com

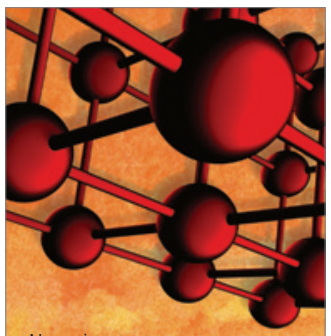

Materials Science and Engineering
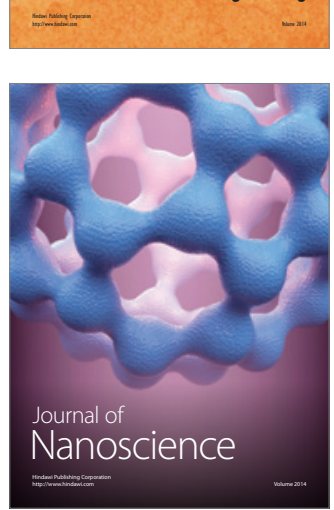
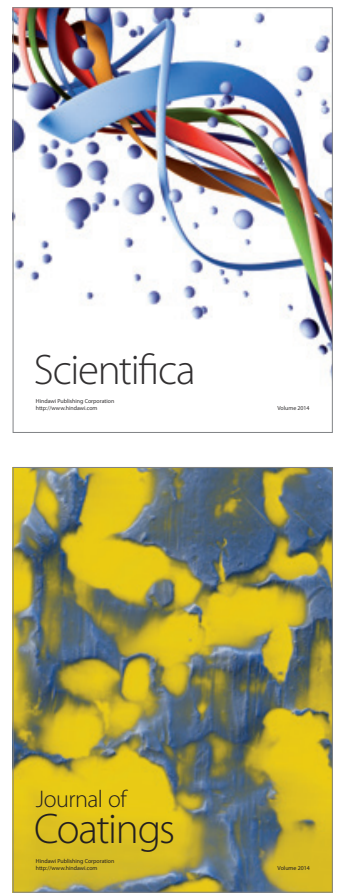
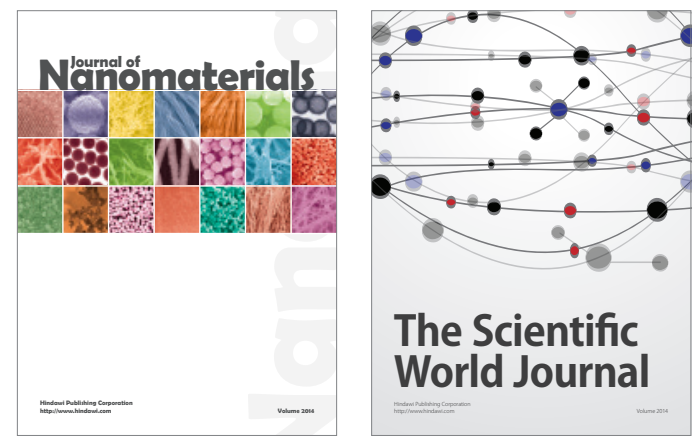

The Scientific World Journal
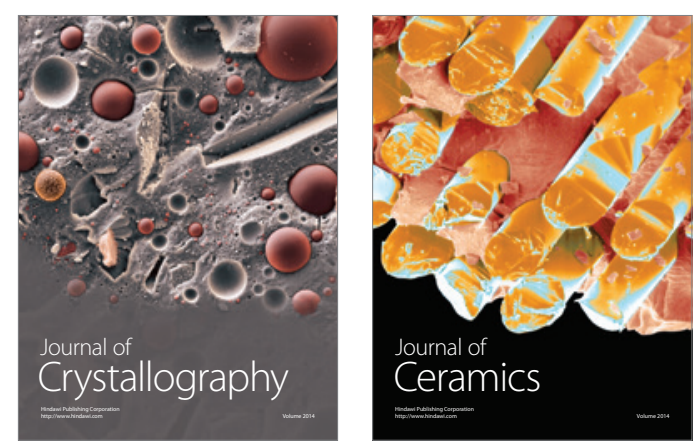
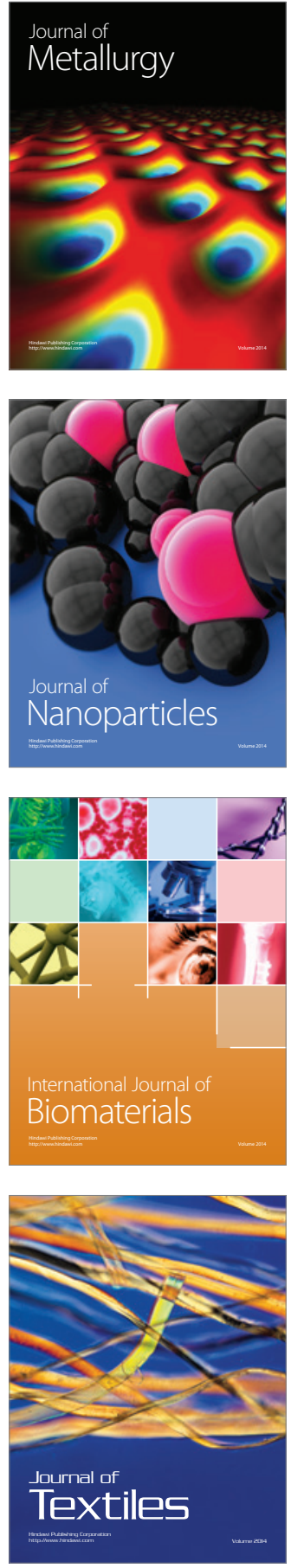